\title{
Comparison of two magnetic resonance imaging spectroscopy postprocessing methods
}

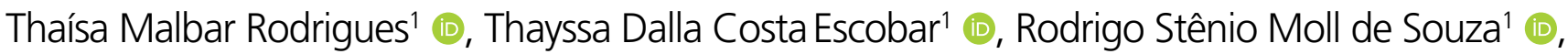 \\ Ester Miyuki Nakamura-Palacios² ${ }^{10}$, Marcos Rosa-Júnior ${ }^{1,3 *}$
}

\begin{abstract}
SUMMARY
OBJECTIVE: The aim of this study was to compare the results obtained using SpectroView ${ }^{\circledR}$ (SV) and J-Magnetic Resonance User Interface (jMRUI) from the same magnetic resonance (MR) spectroscopy of hydrogen data.

METHODS: Data from 23 males with alcohol use disorder (AUD) and 23 healthy non-AUD males were acquired by a 1.5 Tesla MR using a PRESS sequence ( $T E=30 \mathrm{~ms}$ ) in four voxels located in the right frontal and left frontal (RF and LF) lobes, and posterior cingulate (AC and PC). The ratio of the signals from both $\mathrm{N}$-acetyl-aspartate (NAA) and choline (Cho) over creatine ( $\mathrm{Cr}$ ) was calculated automatically using SV and semiautomatically by an expert neuroradiologist using jMRUI. The software' agreement was calculated by the $95 \%$ limits of agreement (LOA) of the ratio of the obtained values.

RESULTS: The standard deviation was greater in jMRUI than in SV. Although there was a correlation between the results from both methods, it was not possible to predict their variance from one another. Additionally, the $95 \%$ LoA showed that jMRUI values were expected to vary from 38 to $190 \%$ of those obtained using SV for NAA/Cr in RF of AUD subjects and from 48 to $196 \%$ for NAA/Cr in CA of non-AUD individuals.

CONCLUSIONS: The difference between the methods may represent clinically significant magnitudes. We suggest the use of the same method when comparing spectroscopic data. We also suggest that in clinical practice, the automatic method should be preferred.

KEYWORDS: Magnetic resonance spectroscopy. Alcoholism.
\end{abstract}

\section{INTRODUCTION}

Proton magnetic resonance spectroscopy $\left({ }^{1} \mathrm{H}-\mathrm{MRS}\right)$ is a noninvasive method, which demonstrates brain tissues' biochemistry ${ }^{1,2}$. Through ${ }^{1} \mathrm{H}-\mathrm{MRS}$, it is possible to identify the wave frequency from hydrogen protons that characterize specific metabolites and their signals' amplitudes, with the latter used to calculate the relative concentration of metabolites ${ }^{3}$. Since creatine $(\mathrm{Cr})$ is the most stable metabolite captured by ${ }^{1} \mathrm{H}-\mathrm{MRS}$ in brain tissue, it is the most adopted as an internal reference to measure the concentration of other metabolites ${ }^{4,5}$.

Although ${ }^{1} \mathrm{H}-\mathrm{MRS}$ does not show specific disease lesions, it demonstrates general metabolic alterations including neuronal viability, the cellular energy status, and the state of cell membrane ${ }^{5}$. Recently, ${ }^{1} \mathrm{H}$-MRS has been used clinically and scientifically to investigate various neurologic alterations, such as metabolic, inflammatory, infectious, and tumoral diseases ${ }^{1,2}$.

\footnotetext{
${ }^{1}$ Universidade Federal do Espírito Santo, Hospital Universitário Cassiano Antônio de Moraes - Vitória (ES), Brazil.

2Universidade Federal do Espírito Santo, Laboratório de Ciências Cognitivas e Neuropsicofarmacologia, Programa de Pós-Graduação em Ciências Fisiológicas - Vitória (ES), Brazil.

${ }^{3}$ Universidade Federal do Espírito Santo, Departamento de Clínica Médica, Centro de Ciências da Saúde - Vitória (ES), Brazil.

*Corresponding author: marcosrosajr@hotmail.com

Conflicts of interest: the authors declare there is no conflicts of interest. Funding: this study was funded by Conselho Nacional de Desenvolvimento Científico e Tecnológico (CNPq).

Received on August 03, 2021. Accepted on August 30, 2021.
} 
The signals of metabolites analyzed can be interfered with by many factors, such as the signal of water and the adjacent structures (e.g., the liquor and the skull) ${ }^{1,6}$. Therefore, the postprocessing of data is imperative in order to suppress noises and identify the metabolites of interest ${ }^{1,5}$. Despite many innovations in data acquiring and processing automatization, there is still a gap between scientific research and clinical application of the ${ }^{1} \mathrm{H}$-MRS, partially due to the complexity of the technique and the manual steps of postprocessing ${ }^{7,8}$.

It is still not much discussed in the literature if the metabolites' ratios in ${ }^{1} \mathrm{H}$-MRS vary in different postprocessing methods. The aim of this study was to compare the results obtained by an automatic (J-magnetic resonance user interface, jMRUI) and a semiautomatic (SpectroView ${ }^{\circledR}$ [SV], Philips Medical Systems Nederland B.V., The Netherlands) postprocessing method of ${ }^{1} \mathrm{H}-\mathrm{MRS}$.

\section{METHODS}

\section{Inclusion and exclusion criteria}

This study is part of a research project that aimed to evaluate spectroscopic differences in the brain of patients with alcohol use disorder (AUD) and healthy non-AUD subjects with the approval of the Local Ethics Committee. Therefore, this study followed the same inclusion and exclusion criteria of the research project, which may be consulted in the article by de
Souza et al. ${ }^{9}$. The recruitment of both groups was performed between March 2016 and July 2017. Those who were eligible and in agreement to participate in this study signed the Informed Consent Form and were submitted to anamnesis, medical record analysis, magnetic resonance imaging (MRI), and MRS-H.

\section{Data acquisition}

${ }^{1} \mathrm{H}-\mathrm{MRS}$ data were acquired by a 1.5 Tesla MRI scanner Achieva (Philips Medical Systems Nederland B.V.), with a specific neurovascular coil, model SENSE Head Coil with eight channels, without paramagnetic contrast administration.

The MR acquisition was realized by point-resolved spectroscopy sequence (PRESS), in the axial plane, with echo-time of $30 \mathrm{~ms}$, repetition-time of $6 \mathrm{~s}$, and NEX of 2 . The total number of acquisitions was 64 , with a bandwidth of $4 \mathrm{kHz}$ and a spectral resolution of 4096 points, without suppression of the water signal. The spectroscopic acquisition was performed in four single voxels, each with $8 \mathrm{~cm}^{3}(2 \mathrm{~cm} \times 2 \mathrm{~cm} \times 2 \mathrm{~cm})$, localized in left dorsolateral prefrontal $(\mathrm{LF})$, right dorsolateral prefrontal $(\mathrm{RF})$, anterior cingulate (AC), and posterior cingulate (PC), including both white and gray matter. The estimated time for each sequence was $2 \mathrm{~min}$ and $40 \mathrm{~s}$ (Figure 1).

\section{Data postprocessing}

Data were processed automatically using SpectroView ${ }^{\circledR}$ (Philips, NL), resulting in ratios of the signals' amplitudes
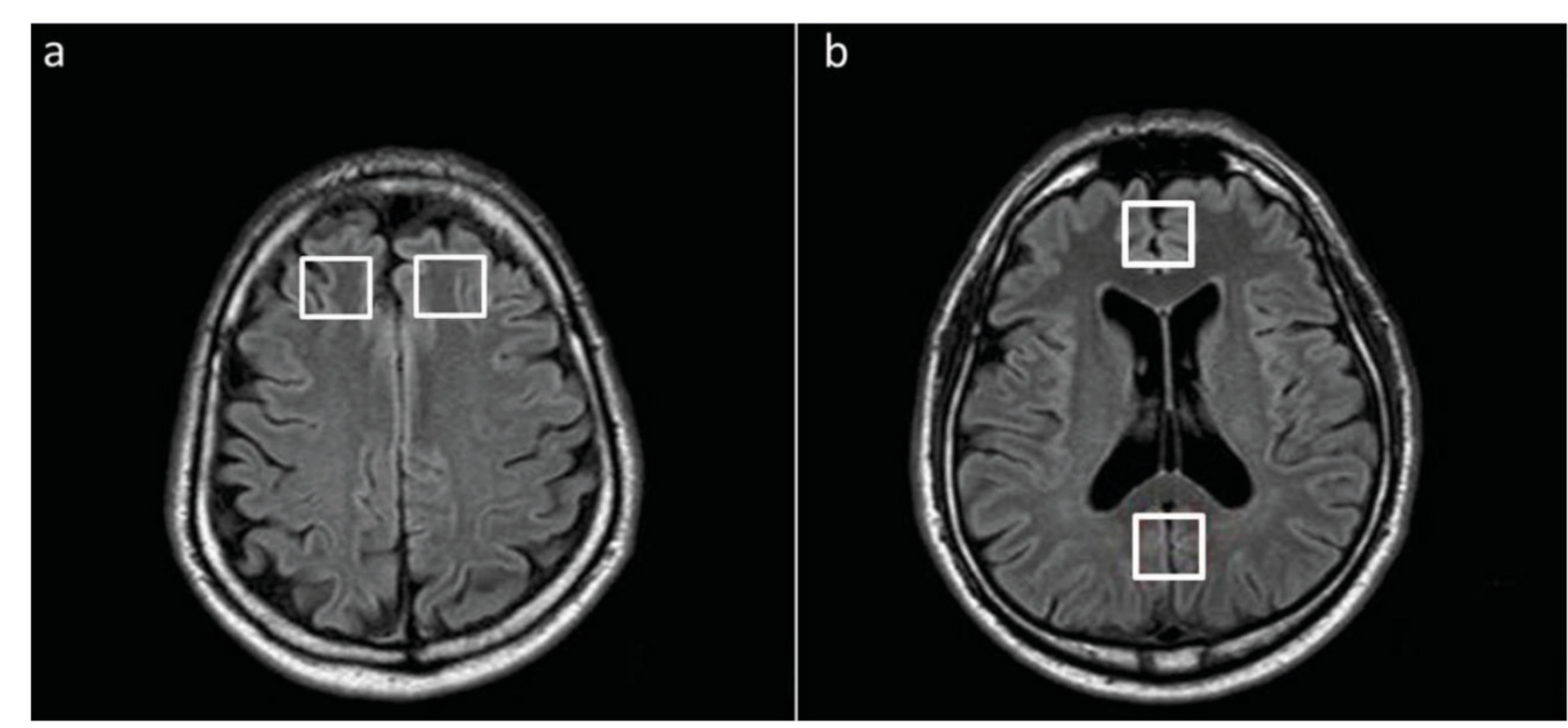

Figure 1. Illustrative magnetic resonance image showing the voxels analyzed $(2 \mathrm{~cm} \times 2 \mathrm{~cm} \times 2 \mathrm{~cm})$ represented in white boxes in (A) (right and left dorsolateral prefrontal) and in (B) (anterior and posterior cingulate). 
from $N$-acetyl-aspartate (NAA) and choline (Cho) over Cr. Subsequently, all data were processed by the same examiner, a neuroradiologist (M.R.J.) with 8 years of experience, through the jMRUI program (public-domain MR user-interface software ${ }^{10,11}$. In the jMRUI analysis, the Advanced Method for Accurate, Robust, and Efficient Spectral fitting (AMARES) algorithm was used to fit the time-domain data ${ }^{12}$, with water peak assigned to $4.68 \mathrm{ppm}, \mathrm{NAA}$ to $2.02 \mathrm{ppm}$, Cr to $3.0 \mathrm{ppm}$, and Cho to $3.2 \mathrm{ppm}$. The spectral curves were classified as good quality or inadequate quality for analysis, based on the baseline characteristics (the quality of water suppression and the quality of the separation of metabolite peaks), as well as the frequency, shape, and amplitude of the identified peaks. Inadequate spectroscopies were excluded from the analysis, due to the low reliability of these results and to the already known greater difference between SV and jMRUI when analyzing bad-quality spectroscopies ${ }^{13}$.

\section{Statistical analysis}

Initially, the metabolites were analyzed descriptively (mean, lower and upper values, and standard deviation). Subsequently, we executed a linear regression analysis with the SV's results introduced as the independent variable and the jMRUI's results as the dependent variable, separately by group, metabolite, and location analyzed.

To identify the agreement between the programs, we applied the adapted method of Bland and Altman plot ${ }^{14}$ as follows: in Y-axis, we located the ratio between the value obtained by jMRUI over the value obtained by SV in each measurement (jMRUI/SV); while in X-axis, we located the mean of the values obtained by the methods in each measurement [(jMRUI+SV)/2].
Then, we calculated and included in this graph the $95 \%$ limits of agreement (LoA; $-1.96 \mathrm{SD}$ and +1.96 SD of jMRUI/SV), which represent the interval in which we expected to find 95\% of the values of the ratio jMRUI/SV.

The statistical analysis was performed using SPSS version 23 software (IBM Corp., USA), and p-value $<0.05$ was considered statistically significant.

\section{RESULTS}

In total, 41 individuals (23 healthy non-AUD subjects and 18 AUD patients) were included in the statistical analysis, after the exclusion of 1 AUD patient due to cerebral lesion and 4 AUD patients who presented spectroscopies with a heterogeneous pattern (low quality). NAA value from AC of one AUD patient and Cho value from CP of one non-AUD subject were also excluded from the analysis due to the low quality of their peaks.

Means and standard deviations obtained by both programs are presented in Table 1. Notably, the standard deviations from jMRUI were greater than those from the SV.

For most of the metabolites, there was a significant correlation between the methods' results $(\mathrm{p}<0.01)$. However, in AUD individuals, we could predict at most $69.6 \%$ of the variance of $\mathrm{NAA} / \mathrm{Cr}$ in FE from $\mathrm{jMRUI}$ with SV as the independent variable $(\mathrm{p}<0.0001 ; 95 \% \mathrm{CI} 0.648-1.348)$, while at most $44.3 \%$ of the variance of $\mathrm{NAA} / \mathrm{Cr}$ in $\mathrm{FE}$ of non-AUD individuals $(\mathrm{p}=0.001 ; 95 \% \mathrm{CI} 0.408-1.253)$. Therefore, although there was a correlation between most of the results, we could not create a linear prediction model between the methods.

Additionally, the 95\% LoA were variables for each metabolite and analyzed regions (Figure 2). Among the analyses, the

Table 1. Metabolites' concentration ratios per voxels' locations.

\begin{tabular}{|c|c|c|c|c|c|c|c|c|c|}
\hline & \multirow{3}{*}{$\begin{array}{c}\text { AUD/Non-AUD } \\
n\end{array}$} & \multicolumn{4}{|c|}{ jMRUI } & \multicolumn{4}{|c|}{ SpectroView $^{\circledR}$} \\
\hline & & \multicolumn{2}{|c|}{ AUD } & \multicolumn{2}{|c|}{ Non-AUD } & \multicolumn{2}{|c|}{ AUD } & \multicolumn{2}{|c|}{ Non-AUD } \\
\hline & & Mean & SD & Mean & SD & Mean & SD & Mean & SD \\
\hline NAA/Cr (LF) & $18 / 23$ & 1.51 & 0.58 & 0.49 & 0.3 & 1.53 & 0.48 & 1.52 & 0.24 \\
\hline Cho/Cr (LF) & $18 / 23$ & 0.9 & 0.21 & 1.09 & 0.33 & 0.96 & 0.18 & 1.01 & 0.08 \\
\hline NAA/Cr (RF) & $18 / 23$ & 1.47 & 0.31 & 1.5 & 0.4 & 1.32 & 0.21 & 1.51 & 0.26 \\
\hline Cho/Cr (RF) & $18 / 23$ & 0.95 & 0.17 & 1.03 & 0.39 & 0.94 & 0.12 & 1.05 & 0.17 \\
\hline $\mathrm{NAA} / \mathrm{Cr}(\mathrm{AC})$ & $17 / 23$ & 1.41 & 0.44 & 1.58 & 0.43 & 1.35 & 0.29 & 1.32 & 0.16 \\
\hline Cho/Cr (AC) & $18 / 23$ & 0.76 & 0.18 & 0.86 & 0.2 & 0.84 & 0.12 & 0.9 & 0.11 \\
\hline $\mathrm{NAA} / \mathrm{Cr}(\mathrm{PC})$ & $18 / 23$ & 1.47 & 0.43 & 1.59 & 0.36 & 1.49 & 0.18 & 1.54 & 0.14 \\
\hline Cho/Cr (PC) & $18 / 22$ & 0.57 & 0.11 & 0.6 & 0.07 & 0.61 & 0.06 & 0.63 & 0.08 \\
\hline
\end{tabular}

jMRUI: J-magnetic resonance user interface; AUD: alcohol used disorder; SD: standard deviation; LF: location of voxels (left frontal); RF: right frontal; AC: anterior cingulate; PC: posterior cingulate. 


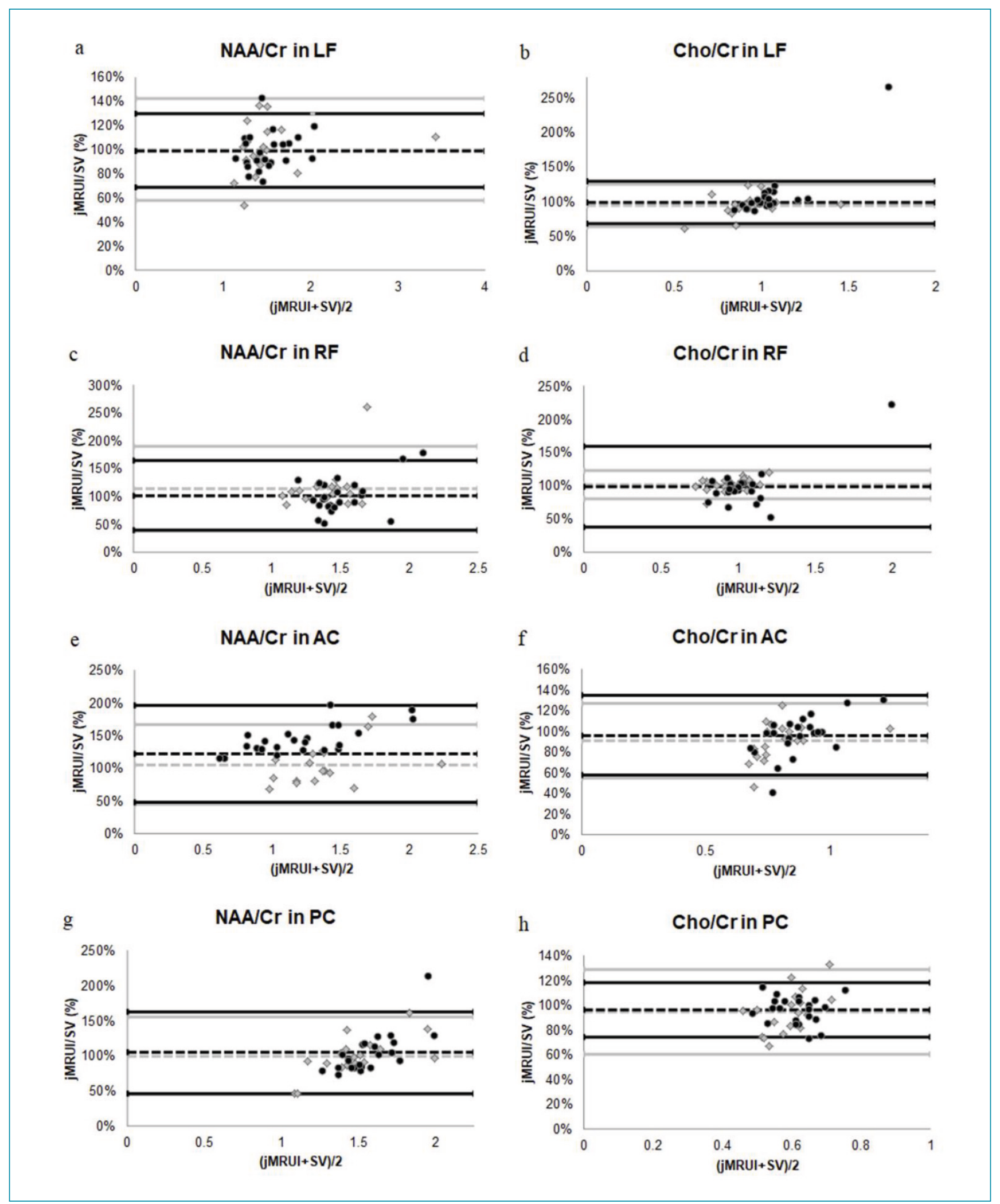

Figure 2. Bland-Altman plots (a-h) showing the ratio of the values obtained by magnetic resonance user interface over the values obtained by SpectroView ${ }^{\circledR}$ (in percentage) in the $Y$-axis and the mean values between the programs in the $X$-axis for each individual. The alcohol use disorder group is represented in gray and the healthy non-alcohol use disorder group in black. Mean values for each group are represented as dashed lines and upper and lower 95\% limits of agreement are represented as solid lines. Location of voxels is represented as left frontal, right frontal, anterior cingulate, and posterior cingulate. 
narrowest LoA were from Cho/Cr in RF from AUD patients (therefore, the most agreeable metabolite), with $95 \%$ of jMRUI measurements' values expected to vary between $80 \%$ and $122 \%$ of SV measurements. In contrast, the broadest LoA were from $\mathrm{NAA} / \mathrm{Cr}$ in RF from AUD patients, with $95 \%$ of the expected results of jMRUI ranging from $38 \%$ to $190 \%$ in comparison with the same measurements obtained by SV.

\section{DISCUSSION}

In this study, we compared the ${ }^{1} \mathrm{H}-\mathrm{MRS}$ postprocessing performed by using an automatic method (SV) and a semiautomatic method (jMRUI), in AUD and non-AUD individuals, since it is expected that different steps followed by each model of postprocessing may result in different measurements of the metabolite ratios ${ }^{1}$. The semiautomatic postprocessing model depends on specialized knowledge and is subjected both to the experience of the examiner and to protocols of different hospitals and research centers ${ }^{13}$. In contrast, the automatic model executes standardized and automatic steps, not depending on a specialized examiner.

Standard deviations of the measurements from the semiautomatic method were greater than those from the automatic method in both AUD and non-AUD groups. This finding supports the previous study by Mazzoni et al. ${ }^{13}$, which demonstrated the same tendency in healthy individuals. In addition, this study showed greater standard deviations in jMRUI also when analyzing patients with expected brain metabolic changes, as demonstrated in studies with AUD patients ${ }^{15,16}$.

Although the methods presented a positive correlation between their results in most metabolites, it was not possible to create a general prediction model to determine the variance of semiautomatic values predicted by automatic results.

Previous studies showed that adjacent structures, such as the liquor and the skull, could influence ${ }^{1} \mathrm{H}$-MRS results. Besides, Mazzoni et al. ${ }^{13}$ verified greater LoA between the programs when evaluating regions closer to the liquor, in comparison with more distant regions, and therefore, they hypothesized that the proximity to the water signal could increase the discordance between the software. In contrast, we did not find such a difference when comparing the LoA of the areas closer to the liquors (i.e., $\mathrm{AC}$ and $\mathrm{PC}$ ) with those more distant from the water signals (i.e., RF and LF).

By analyzing the LoA, we estimated that $95 \%$ of $\mathrm{jMRUI}$ measurements expected for NAA/Cr in RF from AUD patients ranged from $38 \%$ to $190 \%$ of the value obtained by SV from the same metabolite, representing a non-negligible difference in the spectroscopic analysis. Illustratively, a previous study by Bulakbasi et al. ${ }^{17}$ analyzing spectroscopic differences from malignant tumor, benign tumor, and control tissues identified a mean value for $\mathrm{NAA} / \mathrm{Cr}$ of $1.05,1.42$, and 1.68 , respectively, being this difference of less than $50 \%$ of NAA/Cr mean between these tissues significant in the differentiation of tumors $(p<0.05)^{18}$. LoA such as these found in our study evidentiate the possible magnitude of difference between the methods and the relevance of adapting reference values to each of them, as well as the importance of specifying the software and the steps of postprocessing adopted in studies.

In spite of the existing studies and data banks that establish reference values ${ }^{18}$, there is no gold-standard spectroscopic postprocessing method defined yet. Therefore, a common practice in research evaluating brain alterations through ${ }^{1} \mathrm{H}$-MRS is the use of control individuals as parameters of comparison, instead of using a predetermined reference value for identifying brain alterations. Similarly, we considered that in clinical practice, it would be useful to create a spectroscopic data bank in each institution (with its standardized hardware and software), in order to facilitate the clinical application of MRS-H.

As a limitation, our sample size limits the statistical comparison between the software. Besides, the use of ratios of NAA and Cho over $\mathrm{Cr}$ does not allow us to determine if the differences in the measurement of metabolites are due to the analysis of Cr, of both NAA and Cho, or of all of them.

\section{CONCLUSIONS}

The analyzed methods presented differences that may be clinically significant in both AUD and non-AUD individuals. Due to the smaller dispersion presented by the results from SV and the facility in the method execution, we suggest that the automatic may be preferred in clinical practice.

\section{ETHICAL APPROVAL}

All procedures performed in studies involving human participants were in accordance with the ethical standards of the institutional and/or national research committee and with the 1964 Helsinki Declaration and its later amendments or comparable ethical standards. Ethical approval was provided by the Conselho Brasileiro de Ética em Pesquisa da Universidade Federal do Espírito Santo (CAAE 19403713.6.0000.5060 and 13528213.2.0000.5060), Brazil.

\section{INFORMED CONSENT}

Informed consent was obtained from all individual participants included in this study. 


\section{AUTHORS" CONTRIBUTIONS}

TMR: Conceptualization, Data curation, Formal analysis, Funding acquisition, Investigation, Methodology, Validation, Visualization, Writing - original draft. TDCE: Conceptualization, Data curation, Formal analysis, Investigation, Methodology, Validation, Visualization. RSMS: Conceptualization, Data curation, Formal analysis, Investigation, Methodology, Validation, Visualization,
Writing - review \& editing. EMNP: Conceptualization, Data curation, Formal analysis, Funding acquisition, Investigation, Methodology, Validation, Visualization, Writing - review \& editing. MRJ: Conceptualization, Data curation, Formal analysis, Funding acquisition, Investigation, Methodology, Project administration, Resources, Software, Supervision, Validation, Visualization, Writing - review \& editing

\section{REFERENCES}

1. Bertholdo D, Watcharakorn A, Castillo M. Brain proton magnetic resonance spectroscopy: introduction and overview. Neuroimaging Clin N Am. 2013;23(3):359-80. https://doi. org/10.1016/j.nic.2012.10.002

2. Drost DJ, Riddle WR, Clarke GD, AAPM MR Task Group \#9. Proton magnetic resonance spectroscopy in the brain: report of AAPM MR Task Group \#9. Med Phys. 2002;29(9):2177-97. https://doi.org/10.1118/1.1501822

3. Gallagher TA, Nemeth AJ, Hacein-Bey L. An introduction to the Fourier transform: relationship to MRI. AJR Am J Roentgenol. 2008;190(5):1396-405. https://doi.org/10.2214/ AJR.07.2874

4. Ramin SL, Tognola WA, Spotti AR. Espectroscopia de prótons por ressonância magnética: aplicações clínicas em pacientes com lesões encefálicas. Sao Paulo Med J. 2003;121(6):254-9. https://doi.org/10.1590/\$1516-31802003000600008

5. Cecil KM. Proton magnetic resonance spectroscopy: technique for the neuroradiologist. Neuroimaging Clin N Am. 2013;23(3):381-92. https://doi.org/10.1016/j. nic.2012.10.003

6. Zhu H, Barker PB. MR spectroscopy and spectroscopic imaging of the brain. Methods Mol Biol. 2011;711:203-26. https:// doi.org/10.1007/978-1-61737-992-5_9

7. Stanley JA. In vivo magnetic resonance spectroscopy and its application to neuropsychiatric disorders. Can J Psychiatry. 2002;47(4):315-26. https://doi. org/10.1177/070674370204700402

8. in 't Zandt $H$, van Der Graaf M, Heerschap A. Common processing of in vivo MR spectra. NMR Biomed. 2001;14(4):22432. https://doi.org/10.1002/nbm.707

9. de Souza RSM, Rosa Jr M, Rodrigues TM, Escobar TDC, Gasparetto EL, Nakamura-Palacios EM. Lower choline rate in the left prefrontal cortex is associated with higher amount of alcohol use in alcohol use disorder. Front Psychiatry. 2018;9:563. https://doi.org/10.3389/fpsyt.2018.00563
10. Naressi A, Couturier C, Devos JM, Janssen M, Mangeat $C$, de Beer $R$, et al. Java-based graphical user interface for the MRUI quantitation package. MAGMA. 2001;12(2-3):141-52. https://doi.org/10.1007/BF02668096

11. Stefan D, Di Cesare F, Andrasescu A, Popa E, Lazariev A, Vescovo $E$, et al. Quantitation of magnetic resonance spectroscopy signals: the jMRUI software package. Measurement Science and Technology. 2009;20(10):104035.

12. Vanhamme L, van den Boogaart A, Van Huffel S. Improved method for accurate and efficient quantification of MRS data with use of prior knowledge. J Magn Reson. 1997;129(1):3543. https://doi.org/10.1006/jmre.1997.1244

13. Mazzoni LN, Belli G, Ginestroni A, Pratesi A, Agnoloni S, Diciott $S$, et al. Computation of brain metabolite ratios in single-voxel proton MR spectroscopy: comparison between semiautomatic and automatic software. Radiol Med. 2010;115(1):125-32. https://doi.org/10.1007/s11547-009-0408-4

14. Bland JM, Altman DG. Measuring agreement in method comparison studies. Stat Methods Med Res. 1999;8(2):13560. https://doi.org/10.1177/096228029900800204

15. Ende G, Walter S, Welzel H, Demirakca T, Wokrina T, Ruf M, et al Alcohol consumption significantly influences the MR signal of frontal choline-containing compounds. Neuroimage. 2006;32(2):740-6. https://doi.org/10.1016/j.neuroimage.2006.03.049

16. Meyerhoff DJ. Brain proton magnetic resonance spectroscopy of alcohol use disorders. Handb Clin Neurol. 2014;125:31337. https://doi.org/10.1016/B978-0-444-62619-6.00019-7

17. Bulakbasi N, Kocaoglu M, Ors F, Tayfun C, Uçöz T. Combination of single-voxel proton MR spectroscopy and apparent diffusion coefficient calculation in the evaluation of common brain tumors. AJNR Am J Neuroradiol. 2003;24(2):225-33. PMID: 12591638

18. Safriel Y, Pol-Rodriguez M, Novotny EJ, Rothman DL, Fulbright RK. Reference values for long echo time MR spectroscopy in healthy adults. AJNR Am J Neuroradiol. 2005;26(6):1439-45. PMID: 15956513 